\author{
ORIGINAL \\ RESEARCH

M.E. Sprengers
J. Schaafsma
W.J. van Rooij
M. Sluzewski
G.J.E. Rinkel
B.K. Velthuis
J.C. van Rijn
C.B. Majoie

\section{Stability of Intracranial Aneurysms Adequately Occluded 6 Months after Coiling: A 3T MR Angiography Multicenter Long-Term Follow-Up Study}

BACKGROUND AND PURPOSE: The long-term fate of coiled intracranial aneurysms is largely unknown, and prolonged imaging follow-up has been advocated. The yield of follow-up imaging in coiled aneurysms adequately occluded at 6 months is unknown. In such patients, we performed time-of-flight MR angiography (MRA) to assess the incidence and therapeutic consequences of reopening 5-11 years after coiling.

\begin{abstract}
MATERIALS AND METHODS: Between 1995 and 2002, 661 aneurysms in 607 patients were coiled in 3 participating centers. Six-month follow-up angiograms were obtained in 497 (75\%) aneurysms, of which 316 (64\%) in 297 patients were adequately occluded. Of 297 patients, 84 were excluded for various reasons and 73 could not be traced. Of 140 eligible patients, 104 (74\%) with 111 aneurysms were studied with 3T MR imaging and high-resolution MRA at a mean of 6.0 years after coiling (median, 5.6 years; range, 5.0-10.6 years)
\end{abstract}

RESULTS: The proportion of aneurysms with reopening was $3.6 \%(4 / 111 ; 95 \%$ confidence interval [Cl], 1.1\%-9.2\%). One reopened aneurysm, which initially contained intraluminal thrombus, was additionally coiled $(0.9 \% ; 95 \% \mathrm{Cl}, 0.0 \%-5.4 \%)$

CONCLUSION: In intracranial aneurysms with adequate occlusion at 6 months after coiling, the proportion of reopening needing retreatment after $>5$ years is low. The number of reopened aneurysms with therapeutic consequences was too small to assess risk factors, but probably the presence of intraluminal thrombus is one such risk factor. Most patients with coiled intracranial aneurysms that are adequately occluded at 6 months might not need prolonged imaging follow-up.
E ndovascular embolization with detachable coils has become an established technique for the treatment of patients with intracranial aneurysms. ${ }^{1,2}$ In approximately $15 \%-$ $40 \%$ of patients, the coiled aneurysm reopens at follow-up due to coil compaction or resolution of intraluminal thrombus. ${ }^{3-13}$ The most important and constantly found risk factors for reopening with time are large aneurysm size, low packing attenuation, initial incomplete occlusion, and the initial presence of intraluminal thrombus. Risk factors in some but not all studies are rupture status, aneurysm location, and neck size. In several studies, more first-time recurrences were found with longer follow-up. ${ }^{4,5,8}$ In contrast, we found in a previous study of 164 aneurysms with angiographic follow-up at fixed intervals of 6 and 18 months that all aneurysm recurrences were apparent at first angiographic follow-up and aneurysms that were adequately occluded at 6 months remained so at the 18 month interval. ${ }^{3}$ We postulate that if this stability of aneurysm occlusion between 6 and 18 months could be confirmed in a longer follow-up study, it could have important consequences for the follow-up strategy of coiled intracranial aneurysms. In

Received February 26, 2008; accepted after revision April 23.

From the Department of Radiology (M.E.S., W.J.v.R., M.S.), St. Elisabeth Ziekenhuis, Tilburg, the Netherlands; Department of Radiology (M.E.S., C.B.M., J.C.v.R.), Academisch Medisch Centrum, Amsterdam, the Netherlands; and Department of Radiology (J.S., B.K.V., G.J.E.R.), Universitair Medisch Centrum, Utrecht, the Netherlands.

This work was supported by grants from the Dutch Brain Foundation, the Nuts Ohra Foundation, and Boston Scientific, Maastricht, the Netherlands.

Please address correspondence to Willem Jan van Rooij, MD, PhD, Department of Radiology, St. Elisabeth Ziekenhuis, Hilvarenbeekseweg 60, 5022 GC Tilburg, the Netherlands; e-mail: radiol@knmg.nl

DOI 10.3174/ajnr.A1181 this long-term follow-up study, we performed 3T MR imaging and MR angiography (MRA), 5-11 years after coiling, in a sample of a large multicenter cohort of patients with aneurysms that were adequately occluded at first angiographic follow-up at 6 months to assess the incidence and therapeutic consequences of long-term reopening.

\section{Materials and Methods}

\section{Patients}

This study was approved by the institutional review board of the 3 participating hospitals in the Netherlands (St. Elisabeth Ziekenhuis, Tilburg; Academisch Medisch Centrum, Amsterdam; and Universitair Medisch Centrum, Utrecht) and written informed patient consent was obtained.

From the data bases of the 3 centers, we selected all patients with a ruptured or unruptured aneurysm coiled in the period from January 1, 1995, to December 31,2002, with adequate aneurysm occlusion (complete occlusion or small neck remnant) at 6-month angiographic follow-up. Further inclusion criteria were current age between 18 and 70 years, independent functional state, and no contraindication for 3T MR imaging. With these criteria, we calculated including approximately 100 patients, the number for which a grant was provided. All aneurysms were treated with Guglielmi detachable coils (Boston Scientific, Fremont, Calif).

We contacted the general practitioners of all eligible patients to find out if the patient was still alive. If a patient had died, we asked for the date and the cause of death. If a patient had died in a hospital or other facility, we reviewed the medical records. The patients who were still alive received a letter with background information and an invitation to participate in this MR imaging follow-up study. Patients 


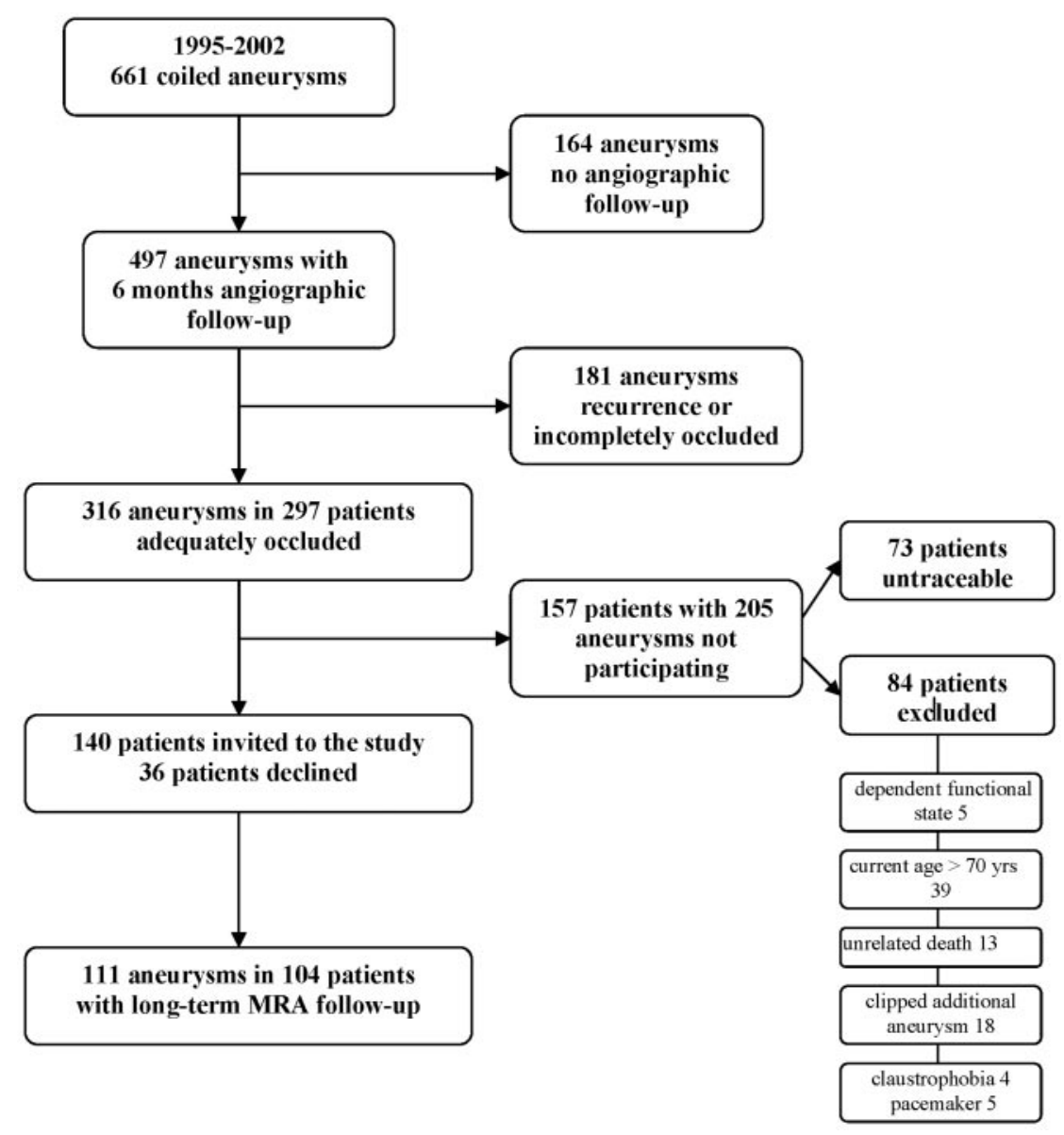

Fig 1. Flow chart of total cohort and included aneurysms.

\begin{tabular}{|c|c|c|c|c|}
\hline Characteristics & $\begin{array}{c}457 \text { Patients with } 497 \text { Aneurysms } \\
\text { with 6-Month Angiographic } \\
\text { Follow-Up }\end{array}$ & $\begin{array}{c}297 \text { Patients with } 316 \text { Aneurysms } \\
\text { with Adequate Occlusion at } 6 \\
\text { Months }\end{array}$ & $\begin{array}{c}36 \text { Patients Fulfilling Inclusion } \\
\text { Criteria Not Participating in } \\
\text { the Study }\end{array}$ & $\begin{array}{l}104 \text { Patients with } 111 \\
\text { Aneurysms with } \\
\text { Long-Term MRA } \\
\text { Follow-Up }\end{array}$ \\
\hline Women & $311(68 \%)$ & 206 (69\%) & 22 (61\%) & $71(69 \%)$ \\
\hline Mean age (yr) & (median, 51; range, 18-82) & 52.0 (median, 51; range, 26-82) & 56.0 (median, 55; range, 36-69) & $\begin{array}{c}54.7 \text { (median, 54; } \\
\text { range, 39-70) }\end{array}$ \\
\hline Ruptured aneurysms & $351(77 \%)$ & $216(72 \%)$ & $30(83 \%)$ & $84(81 \%)$ \\
\hline Aneurysm size (mm) & 2-55 (mean, 9.2; median, 7) & 2-22 (mean, 7.4; median, 6) & 3-15 (mean, 7.4; median, 7) & $\begin{array}{l}\text { 3-17 (mean, 6.6; } \\
\text { median, 5) }\end{array}$ \\
\hline \multicolumn{5}{|l|}{ Aneurysm location } \\
\hline ACA & $137(27.6 \%)$ & $86(27.2 \%)$ & $8(22.2 \%)$ & $23(20.7 \%)$ \\
\hline MCA & $34(6.8 \%)$ & $20(6.3 \%)$ & $1(2.7 \%)$ & $11(9.9 \%)$ \\
\hline Posterior circulation & $167(33.6 \%)$ & $107(33.9 \%)$ & $10(27.7 \%)$ & $46(41.5)$ \\
\hline ICA & $159(32 \%)$ & $103(32.6 \%)$ & $17(47.2 \%)$ & $31(27.9 \%)$ \\
\hline
\end{tabular}

Note:-ACA indicates anterior cerebral artery; MCA, middle cerebral artery; ICA, internal carotid artery.

who consented were invited for MR imaging. A written report of the MR imaging findings was sent to the general practitioner, and patients were informed about results by telephone or at the outpatient clinic.

\section{MR Imaging and MRA Follow-Up Protocol}

MR imaging examinations were performed on a $3 \mathrm{~T}$ system (Intera R10; Philips Medical Systems, Best, the Netherlands) by using the sensitivity encoding (SENSE) phased-array head coil (MR Imaging Devices, Gainesville, Fla). MR imaging protocol included axial T2weighted fast spin-echo and multiple overlapping thin-slab acquisition 3D time-of-flight (MOTSA 3D-TOF) MRA sequences. Imaging parameters for the T2-weighted fast spin-echo sequence were the following: TR/TE, 3394/80 ms; $400 \times 400$ matrix (reconstructed to $512 \times 512$ ); FOV, 230-mm; 70\% rectangular FOV; 5-mm-thick sections with a $0.5-\mathrm{mm}$ gap. The volume of the MOTSA 3D-TOF MRA was localized on a sagittal 2D phase-contrast scout image. A presaturation band was applied above the imaging volume to saturate incoming venous blood. For the MOTSA 3D-TOF MR imaging, the parameters were the following: a $3 \mathrm{D}$ fast-field echo T1-weighted sequence; TR/TE, 21/4 ms; flip angle, $20^{\circ}$; matrix, $512 \times 512$ (reconstructed to $1024 \times 1024)$; FOV, $200 \mathrm{~mm}$; 85\% rectangular FOV; 1.0-mm thick sections, interpolated to $0.5 \mathrm{~mm} ; 160$ sections acquired in 8 chunks. 
The measured voxel size of the MOTSA 3D-TOF MR image was $0.39 \times 0.61 \times 1 \mathrm{~mm}$, and the reconstructed voxel size was $0.2 \times 0.2 \times$ $0.5 \mathrm{~mm}$. The acquisition time of the high-resolution MOTSA 3DTOF sequence was reduced by SENSE parallel imaging. Total MR imaging examination time was 20 minutes. The usefulness of this $3 \mathrm{~T}$ MRA protocol in the follow-up of coiled intracranial aneurysms was validated in a previous study. ${ }^{14}$

\section{MR Imaging and MRA Evaluation}

MRA findings (axial source images, maximum intensity projections, and volume-rendered images) were evaluated and, if necessary, compared with results of angiographic follow-up at 6 months by 2 experienced neuroradiologists independently. Discrepancies were resolved in consensus. Aneurysm-occlusion status was dichotomized as adequate (complete or small neck remnant) or incomplete. Incomplete occlusion was considered as a recurrence, and residual aneurysm was measured in 2 directions. The presence or absence of intraluminal thrombus was assessed on the axial T2-weighted images.

\section{Statistical Analysis}

Interobserver variability for the MRA evaluation (adequate occlusion versus incomplete occlusion) was assessed with $\kappa$ statistics. ${ }^{15}$

Proportions with 95\% confidence intervals (CIs) were calculated for aneurysms with reopening and for aneurysms with retreatment. Participating patients were compared with the remaining eligible patients and with all patients with adequately occluded aneurysms at 6-month follow-up for the following patient and aneurysm characteristics: sex, mean age, proportion of ruptured aneurysms, mean aneurysm size, and aneurysm location classified into 4 categories: anterior cerebral artery, middle cerebral artery, internal carotid artery, and posterior circulation. The $t$ test was used for comparison of means, and the $\chi^{2}$ test was used for comparison of proportions

\section{Results}

\section{Patients}

Between January 1, 1995, and December 31, 2002, 666 aneurysms in 612 patients were coiled in the 3 participating centers (Tilburg, 483 [76\%]; Utrecht, 105 [16\%]; and Amsterdam, 24 [8\%]). Of 612 patients, 457 with 497 aneurysms had 6-month follow-up angiography, and 316 aneurysms in 297 patients were adequately occluded at this first angiographic follow-up (Fig 1). Of 297 eligible patients with 316 aneurysms, 84 were excluded for the following reasons: dependent functional state in 5, age older than 70 years in 39, 3T MR imaging contraindication in 27 (clipped additional aneurysms in 18, claustrophobia in 4, and a pacemaker in 5), unrelated death in 13 (cancer in 6, cardiovascular disease in 3, old age in 1, and unknown but unlikely subarachnoid hemorrhage in 3). Seventy-three patients could not be traced, but many of these patients had previous clinical or angiographic follow-up beyond the 6-month interval. The remaining 140 patients were invited to participate in the study, of whom 36 declined. Thus, 104 patients (Tilburg, 74 [71\%]; Utrecht, 25 [24\%]; and Amsterdam, $5[5 \%])$ with 111 aneurysms participated in this study. Patient-participation grade was 74\% (104/140).

Patient and aneurysm characteristics of all patients with 6-month angiographic follow up, the cohort of patients with adequate aneurysm occlusion at 6 months, the nonparticipat-

\begin{tabular}{ll}
\hline Table 2: Detailed characteristics of $\mathbf{1 0 4}$ patients with $\mathbf{1 1 1}$ \\
aneurysms with long-term MRA follow-up \\
\hline Characteristics \\
\hline Patient \\
Women \\
Mean age (yr) & 71 (69 \%) \\
Aneurysm & 54.7 (median, 54; range, 39-70) \\
Ruptured & \\
Mean size (mm) & 84 (81\%) \\
$\leq 5$ mm & 6.6 (median, 5; range, 3-17) \\
$6-10$ mm & 42 (46.9\%) \\
$\geq 10$ mm & $40(36.1 \%)$ \\
Aneurysm location & $19(17 \%)$ \\
Anterior circulation & \\
Pericallosal artery & 65 (58.5\%) \\
Anterior communicating artery & 3 \\
A1 & 18 \\
Middle cerebral artery & 2 \\
Supraclinoid internal carotid artery & 11 \\
Anterior choroidal artery & 7 \\
Posterior communicating artery & 4 \\
Carotid ophthalmic artery & 14 \\
Posterior circulation & 6 \\
Basilar tip & 46 (41.5\%) \\
Posterior inferior cerebellar artery & 24 \\
Superior cerebellar artery & 10 \\
Posterior cerebral artery & 7 \\
Basilar trunk & 4 \\
\hline
\end{tabular}

ing eligible patients, and the participating patients are displayed in Table 1.

The mean MR imaging follow-up period of 104 participating patients with 111 aneurysms was 6.0 years (median, 5.6 years; range, 5.0-10.6 years; 646 patient years). Patient and aneurysm characteristics of the 104 patients with 111 aneurysms are displayed in Table 2. Of 104 patients, 87 had angiographic follow-up beyond the 6-month follow-up interval.

\section{MR Imaging and MRA Findings}

Of 111 aneurysms in 104 patients, 4 aneurysms (3.6\%; 95\% CI, $1.1 \%-9.2 \%)$ in 4 patients showed reopening on follow-up MRA. One reopened aneurysm was additionally coiled (0.9\%; 95\% CI, 0.0\%-5.4\%). The remaining 107 aneurysms had unchanged adequate occlusion status. There were no discrepancies of long-term MRA with angiographic follow-up results beyond the 6-month follow-up interval. Interobserver agreement for aneurysm occlusion status on follow-up MRA was very good $(\kappa=0.82 ; 95 \% \mathrm{CI}, 0.74-0.90)$, with agreement in $104(94 \%)$ of 111 aneurysms.

The first patient with a recurrence was a 61-year-old woman with a coiled ruptured 3-mm middle cerebral artery aneurysm (Fig 2). MRA at 7.3 years showed a $1 \times 2 \mathrm{~mm}$ intraluminal recurrence, inside the coil mesh. Additional treatment was judged not necessary. The second patient was a 54year-old woman with a coiled ruptured 5-mm basilar tip aneurysm (Fig 3). MRA follow-up at 8.0 years showed a $2 \times 3$ $\mathrm{mm}$ disklike reopening of the base of the aneurysm, unchanged with a 5.8-year follow-up angiogram. Additional treatment was judged not necessary.

The third patient was a 49-year-old woman with a coiled ruptured 10-mm basilar tip aneurysm (Fig 4). A small bleb at the base of the aneurysm could not be occluded with coils. 

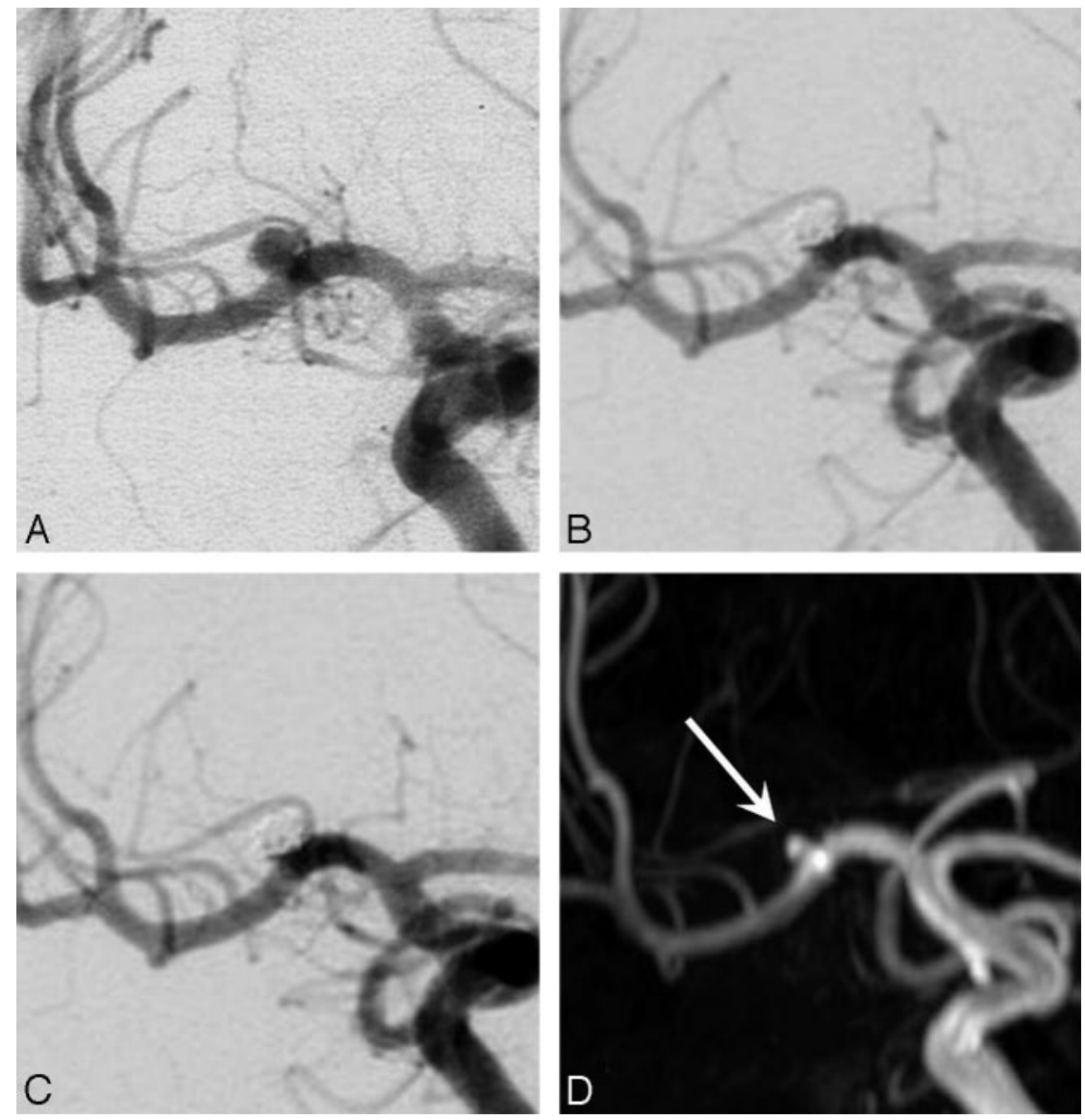

Fig 2. A 61-year-old woman with a ruptured 3-mm middle cerebral artery aneurysm. $A$, Precoiling angiogram shows a small aneurysm. $B$, Complete occlusion after coiling. $C$, Stable complete occlusion at 6 months. D, MRA at 7.3 years demonstrates a $1 \times 2 \mathrm{~mm}$ intraluminal opening inside the coil mesh classified as a recurrence (arrow).

MRA follow-up at 8.1 years showed a $3 \times 5 \mathrm{~mm}$ recurrence at the base of the aneurysm, apparently a regrowth from the initially unoccluded bleb. This regrowth was already present on a 5.5-year follow-up angiogram. Additional treatment was judged not necessary.

The fourth patient was a 66 -year-old man with a coiled unruptured 8-mm middle cerebral artery aneurysm (Fig 5). MR imaging and MRA follow-up at 6.6 years showed a $6 \times 4$ $\mathrm{mm}$ reopening and a small perianeurysmal cyst. Additional coiling was performed. In retrospect, intraluminal thrombus was present on the initial CT scan (Fig 5I); and the aneurysm diameter was $20 \mathrm{~mm}$, with a luminal size of $8 \mathrm{~mm}$. Although follow-up angiography at 6 and 18 months demonstrated complete occlusion, this intraluminal clot had apparently resolved later, resulting in aneurysm reopening. Six months after additional coiling, the aneurysm had reopened again and was coiled for a third time. None of the remaining 110 aneurysms showed intraluminal thrombus on follow-up T2weighted MR images.

\section{Differences between Participating and Nonparticipating Eligible Patients}

The mean size of 111 aneurysms with MRA follow-up was significantly smaller than the mean size of 395 remaining aneurysms with angiographic follow-up at 6 months $(9.6 \pm 6.8$ mm versus $6.8 \pm 3.2 \mathrm{~mm}, P<.0001$ ), but it did not differ from the mean size of $7.6 \pm 4.3 \mathrm{~mm}$ of 225 remaining aneurysms that were adequately occluded at 6 months $(P=.082)$. All other patient and aneurysm characteristics were not significantly different.

\section{Discussion}

Most coiled intracranial aneurysms that are adequately occluded at first angiographic follow-up at 6 months remain occluded during the following 5-11 years. Late follow-up imaging resulted in additional treatment in only $1 \%$ of patients. The 1 aneurysm in which additional treatment was performed had luminal enlargement as a result of the resolution of initially present intraluminal thrombus. Resolution of intraluminal thrombus is a well-known risk factor for reopening with time, ${ }^{10-12}$ and partially thrombosed aneurysms should, therefore, be followed for many years, even when occlusion is stable on 6-month follow-up angiography. The other 3 recurrences at long-term MRA follow-up were small, and retreatment was judged not necessary. One of these 3 recurrences was centrally located in the coil mesh. Central openings are readily apparent on MRA but may not be well-appreciated on angiography due to obscuring peripherally located coils ("helmet" sign). Possibly, this opening was already present from the beginning and should not have been classified as a recurrence.

Because reopening long-term after coiling in the studied subset of aneurysms is rare, risk of primary or recurrent hemorrhage from the coiled aneurysm is extremely low. Thus, extended follow-up imaging during 5-11 years in aneurysms 

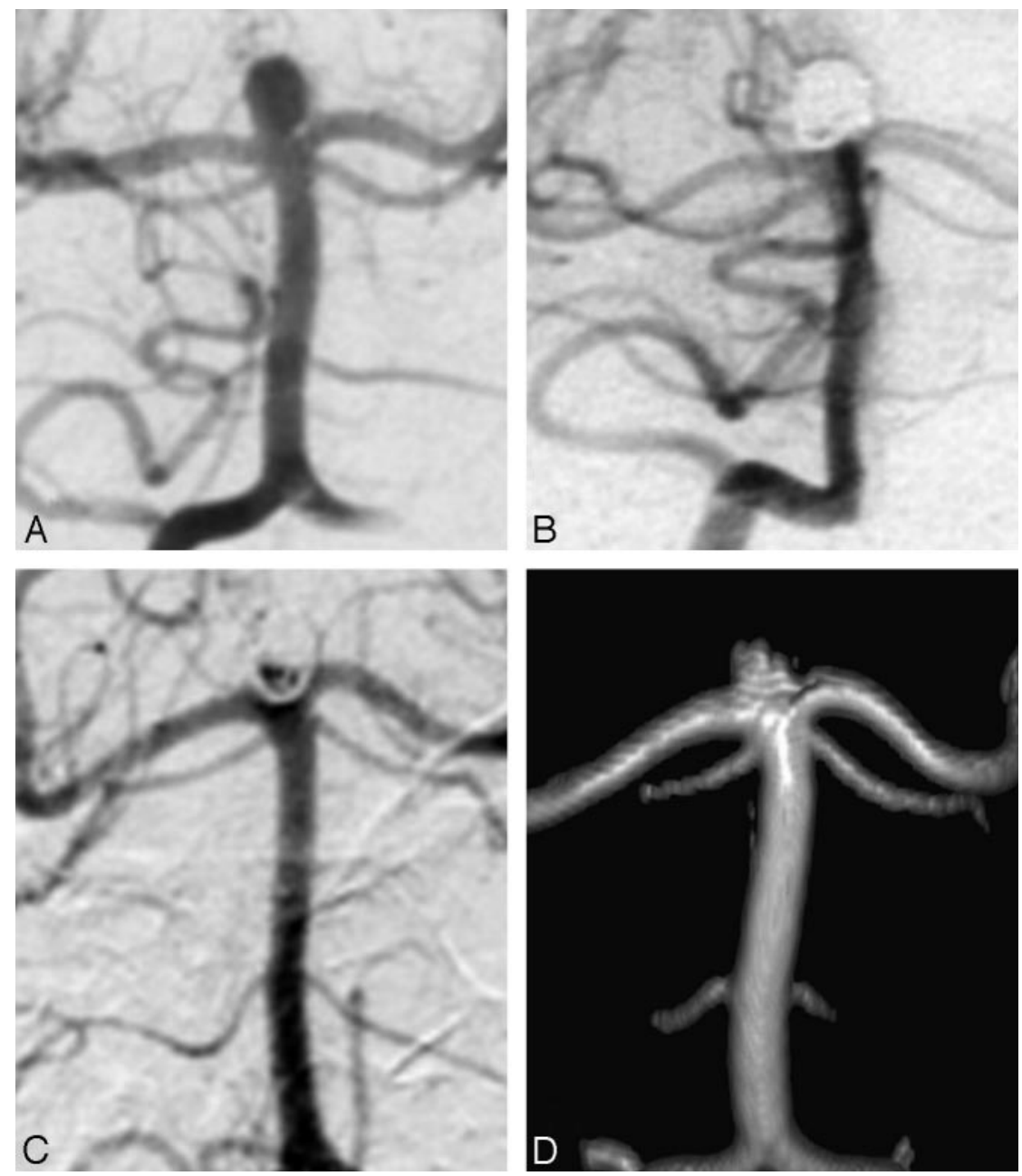

Fig 3. A 54-year-old woman with a ruptured 5 -mm basilar tip aneurysm. $A$, Precoiling vertebral angiogram shows a basilar tip aneurysm. $B$, Stable complete occlusion at 6 months. $C$, Angiogram at 5.8 years shows a $2 \times 3 \mathrm{~mm}$ disklike recurrence at the base of the aneurysm. D, MRA at 8.0 years is unchanged, compared with the last angiogram.

that are adequately occluded at 6 months has within the initial 10 years a low yield in identifying aneurysms that need additional treatment. More research in larger patient groups is needed to identify risk factors for first-time reopening beyond the 6-month interval. Probably, presence of intraluminal thrombus is one such risk factor, and imaging techniques that reliably depict intraluminal thrombus in large and small ruptured and unruptured aneurysms are needed.

This study describes imaging follow-up during the initial 10 years after coiling in a representative sample of a large cohort of patients with stable occlusion at initial follow-up. We could not find other studies on such cohorts to compare with our data. Longer follow-up data (10-20 years) are not yet available from coiled intracranial aneurysms. In a recent study of 756 patients with clipped ruptured aneurysms, the cumulative incidence of recurrent subarachnoid hemorrhage in the first 10 years after clipping was 3.2\%. ${ }^{16}$ Most recurrent hemorrhages were from newly developed aneurysms or untreated additional aneurysms. This indicates that the risk of recurrent hemorrhage from new or existing additional aneurysms in the first 10 years after treatment may be higher than the risk of recurrent hemorrhage from a coiled ruptured aneurysm that is adequately occluded at 6 months. Whether patients with treated aneurysms should be screened for new aneurysms and growth of untreated additional aneurysms after some years is an unresolved issue.

In a previous study with fixed angiographic follow-up intervals of 6 and 18 months, all aneurysm recurrences were apparent on first follow-up, and the benefit of extended angiographic follow-up of adequately occluded aneurysms at 6 months was very low. ${ }^{3}$ Our MRA study confirms this finding at long-term follow-up. In several studies, more first-time aneurysm recurrences after coiling were found with longer follow-up during the first years after coiling. ${ }^{4,5,8}$ However, these studies were characterized by a wide variety of angiographic follow-up intervals, ${ }^{4,8}$ the definition of the initial result partly on 6-month follow-up angiograms, ${ }^{5}$ and aneurysm categorization for comparison based on the length of follow-up without adjustment for other variables influencing reopening. ${ }^{4,5,8}$ In a recent study evaluating retreatment of ruptured aneurysms of International Subarachnoid Aneurysm Trial, ${ }^{13}$ late retreatment was used as a surrogate for aneurysm recurrence. Aneurysm recurrences that were not retreated were not included. The timing of repeat coiling was more or less evenly distributed throughout the follow-up period of $\leq 80$ months; therefore, it was concluded that short-term follow-up imaging is insufficient to detect all recurrences after coiling. However, in this study, the onset of recurrences that were retreated was 

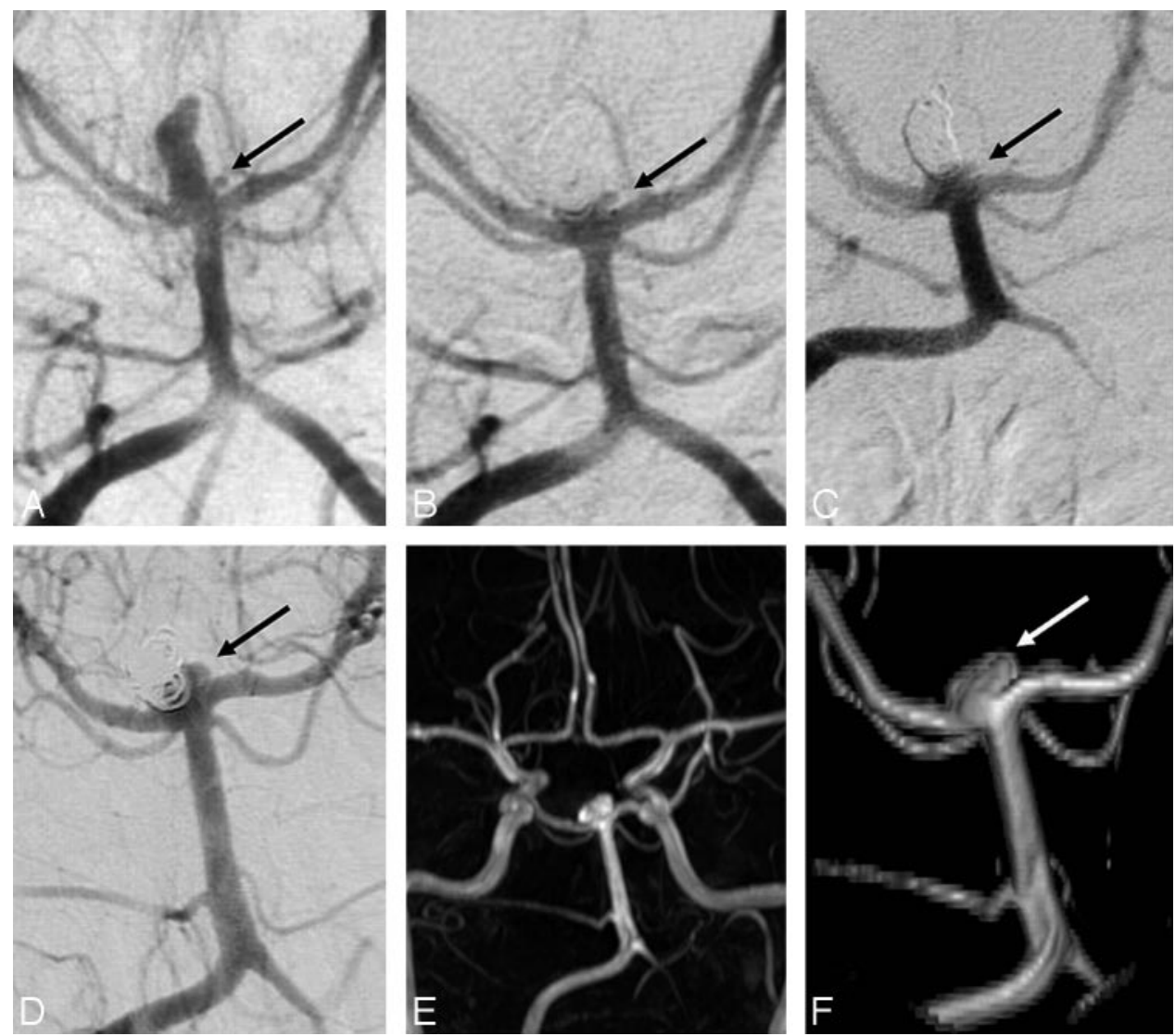

Fig 4. A 49-year-old woman with a ruptured $10-\mathrm{mm}$ basilar tip aneurysm. $A$, Precoiling vertebral angiogram demonstrates the basilar tip aneurysm with a small bleb on its base (arrow) $B$, On the angiogram immediately postcoiling, the bleb (arrow) is not occluded. $C$, Unchanged open bleb (arrow) at 6 months. $D$, Angiogram at 5.5 years with recurrence (arrow) at the base from the bleb. $E$ and $F$, MRA overview $(E)$ and detail $(F)$ at 8.1 years demonstrate unchanged recurrence (arrow).
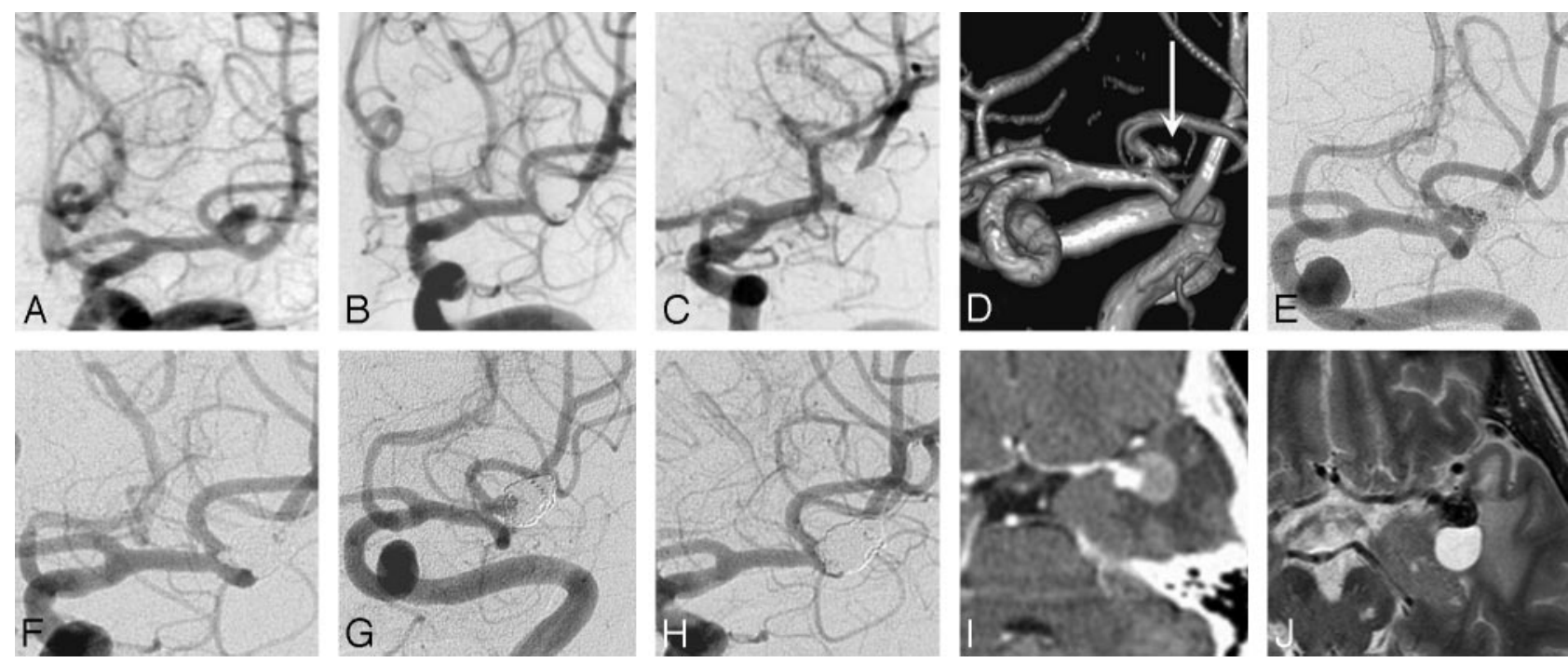

Fig 5. A 66-year-old man presenting with seizures with an unruptured left middle cerebral artery aneurysm. $A$, Precoiling angiogram. $B$, Complete occlusion after coiling. $C$, Stable complete occlusion at 6 months. $D$, MRA at 6.6 years shows a $6 \times 4 \mathrm{~mm}$ recurrence (arrow). $E$, Angiogram before the second coiling. $F$, After the second coiling, again complete occlusion is seen $G$, Six months after the second coiling, repeat reopening of the aneurysm is noted. $H$, After the third coiling, again complete occlusion is seen. Note that the coil mesh is much larger than the aneurysmal lumen in $A$. I, Initial CT angiography before the first coiling demonstrates thrombus surrounding the aneurysm lumen and perifocal edema. J, MR imaging at 6.6 years shows migration of the coil mesh in the thrombosed part of aneurysm and a perianeurysmal cyst.

not evaluated, and the proportion of retreated recurrences already present at 6 months was unknown. Thus, results of these studies are not in contradiction with the finding that virtually all first-time recurrences will be present at 6-month follow-up angiography.
A limitation of our study is that only a portion (35\%) of aneurysms with adequate occlusion at 6 months was evaluated. However, almost three quarters of the patients fulfilling inclusion criteria participated. Patients who could not be included because they were untraceable for this follow-up study 
had at least a 6-month clinical follow-up, and many had follow-up beyond the 6-month interval. We have no reason to believe that the sample was biased because patient and aneurysm characteristics of participants and nonparticipants were not significantly different. Another limitation is the definition of adequate occlusion at 6 months, the main inclusion criterion. Angiographic occlusion status is, to some extent, subjective, especially in aneurysms with neck remnants. In an attempt to limit inclusion bias, we dichotomized between adequately occluded aneurysms and incompletely occluded aneurysms. ${ }^{17}$ Because of the somewhat subjective assessment of adequate versus incomplete occlusion, inconsistencies in inclusion cannot be ruled out completely. However, interobserver agreement for MRA evaluation was very good. Because we have no reason to believe that interobserver variation would be different for digital subtraction angiography than for MRA, inconsistencies in inclusion will have occurred rarely, if at all.

On the basis of present available data, we suggest the following imaging follow-up strategy after coiling: In aneurysms that are adequately occluded 6 months after coiling, no extended imaging follow-up is required (except in partially thrombosed aneurysms and in large and giant aneurysms). In this study, these aneurysms accounted for almost two thirds of all coiled aneurysms. Aneurysms that show reopening (for the most part large and giant aneurysms, initially incompletely occluded aneurysms, and aneurysms that contain intraluminal thrombus) are additionally treated when possible and followed with MRA at regular intervals, because recurrent reopening may occur many years later, even after stable occlusion during some years. All aneurysms $>15 \mathrm{~mm}$ and all aneurysms with intraluminal thrombus are followed on a regular basis, despite complete occlusion at 6 months. ${ }^{9,11,12}$ In our opinion, high-quality 3T MRA (and not conventional angiography) is the preferred imaging technique for follow-up of coiled intracranial aneurysms. ${ }^{14}$ Besides increased safety and better patient compliance, the presence of central aneurysm reopening can be better appreciated and the presence of intraluminal thrombus is readily apparent. An additional advantage is the simultaneous imaging of all cerebral vessels, which allows detection of newly developed aneurysms and growth of additional untreated aneurysms with time.

\section{Conclusion}

In intracranial aneurysms with adequate occlusion at 6 months after coiling, the yield of reopening needing retreat- ment in the next 5-11 years may be low. The number of reopened aneurysms with therapeutic consequences was too small to formally assess risk factors, but the findings suggest that the presence of intraluminal thrombus is one such risk factor.

\section{References}

1. Molyneux AJ, Kerr RS, Yu LM, et al. International Subarachnoid Aneurysm Trial (ISAT) Collaborative Group: International Subarachnoid Aneurysm Trial (ISAT) of neurosurgical clipping versus endovascular coiling in 2143 patients with ruptured intracranial aneurysms - a randomised trial. Lancet 2002;360:1267-74

2. Brilstra EH, Rinkel GJ, van der Graaf Y, et al. Treatment of intracranial aneurysms by embolization with coils: a systematic review. Stroke 1999;30:470-76

3. Sluzewski M, van Rooij WJ, Rinkel GJ, et al. Endovascular treatment of ruptured intracranial aneurysms with detachable coils: long-term clinical and serial angiographic results. Radiology 2003;227:720-24

4. Cognard C, Weill A, Spelle L, et al. Long-term angiographic follow-up of 169 intracranial aneurysms occluded with detachable coils. Radiology 1999;212:348-56

5. Raymond J, Guilbert F, Weill A, et al. Long-term angiographic recurrences after selective endovascular treatment of aneurysms with detachable coils. Stroke 2003;34:1398-403

6. Henkes H, Fischer S, Liebig T, et al. Repeated endovascular coil occlusion in $\mathbf{3 5 0}$ of $\mathbf{2 7 5 9}$ intracranial aneurysms: safety and effectiveness aspects. Neurosurgery 2006;58:224-32

7. Sluzewski M, van Rooij WJ, Slob MJ, et al. Relation between aneurysm volume, packing, and compaction in 145 cerebral aneurysms treated with coils. Radiology 2004;231:653-58

8. Piotin M, Spelle L, Mounayer C, et al. Intracranial aneurysms: treatment with bare platinum coils - aneurysm packing, complex coils, and angiographic recurrence. Radiology 2007;243:500-08. Epub 2007 Feb 9

9. Sluzewski M, Menovsky T, van Rooij WJ, et al. Coiling of very large or gian cerebral aneurysms: long-term clinical and serial angiographic results. AJNR Am J Neuroradiol 2003;24:257-62

10. Slob MJ, Sluzewski M, van Rooij WJ. The relation between packing and reopening in coiled intracranial aneurysms: a prospective study. Neuroradiology 2005;47:942-45

11. Slob MJ, Sluzewski M, van Rooij WJ, et al. Additional coiling of previously coiled cerebral aneurysms: clinical and angiographic results. AJNR Am J Neuroradiol 2004;25:1373-76

12. van Rooij WJ, Sprengers ME, Sluzewski M, et al. Intracranial aneurysms that repeatedly reopen over time after coiling: imaging characteristics and treatment outcome. Neuroradiology 2007;49:343-49

13. Campi A, Ramzi N, Molyneux AJ, et al. Retreatment of ruptured cerebral aneurysms in patients randomized by coiling or clipping in the International Subarachnoid Aneurysm Trial (ISAT). Stroke 2007;38:1538-44

14. Majoie CB, Sprengers ME, van Rooij WJ, et al. MR angiography at 3 T versus digital subtraction angiography in the follow-up of intracranial aneurysms treated with detachable coils. AJNR Am J Neuroradiol 2005;26:1349-56

15. Cohen J. A coefficient of agreement for nominal scales. Educ Psychol Measurem 1960;20:37-46

16. Wermer MJ, Greebe P, Algra A, et al. Incidence of recurrent subarachnoid hemorrhage after clipping for ruptured intracranial aneurysms. Stroke 2005;36:2394-99

17. Cloft HJ, Kaufmann T, Kallmes DF. Observer agreement in the assessment of endovascular aneurysm therapy and aneurysm recurrence. AJNR Am J Neuroradiol 2007;28:497-500 